\title{
Precession missile feature extraction using sparse component analysis of radar measurements
}

\author{
Lihua Liu ${ }^{1 *}$, Xiaoyong Du', Mounir Ghogho ${ }^{2,3}$, Weidong $\mathrm{Hu}^{1}$ and Des McLernon ${ }^{2}$
}

\begin{abstract}
According to the working mode of the ballistic missile warning radar (BMWR), the radar return from the BMWR is usually sparse. To recognize and identify the warhead, it is necessary to extract the precession frequency and the locations of the scattering centers of the missile. This article first analyzes the radar signal model of the precessing conical missile during flight and develops the sparse dictionary which is parameterized by the unknown precession frequency. Based on the sparse dictionary, the sparse signal model is then established. A nonlinear least square estimation is first applied to roughly extract the precession frequency in the sparse dictionary. Based on the time segmented radar signal, a sparse component analysis method using the orthogonal matching pursuit algorithm is then proposed to jointly estimate the precession frequency and the scattering centers of the missile. Simulation results illustrate the validity of the proposed method.
\end{abstract}

\section{Introduction}

Interception of separating ballistic missiles is particularly difficult because the sensor has to be able to discriminate countermeasures, light and heavy decoys, from the warheads within a very limited time [1]. There are three phases in the trajectory of the ballistic missile (BM): boost phase, mid-course phase, and the reentry phase. Mid-course phase is the longest of the three and is the preferred intercept phase in the ballistic missile defense (BMD) system [2].

Since many warheads are spin-stabilized in the midcourse phase, they will precess due to the separation disturbance, and will keep the precession motion until they re-enter the atmosphere [3]. Precession motion, which is a kind of micro-Doppler motion [4], will impose a micro-Doppler modulation effect on the radar echoes, and this is a unique feature of ballistic targets. The precession frequency is an important feature parameter in ballistic target recognition, and it can reflect kinematical characteristics as well as structural and mass distribution features.

At present, the radar based feature extraction for the $\mathrm{BM}$ target recognition mainly includes the following techniques: (1) Electromagnetic scattering feature

\footnotetext{
*Correspondence: gogonudt@126.com

'College of Electronic Science and Engineering, National University of

Defense Technology, Changsha, Hunan 410073, P.R. China

Full list of author information is available at the end of the article
}

extraction, i.e. radar signal amplitude, phase information, and polarization features; (2) Motion feature extraction, i.e. the spinning and precession frequency extraction based on the time-frequency analysis $[5,6]$; (3) Target geometrical structure extraction based on the high resolution range profile (HRRP), ISAR image or three dimensional imaging [7-9].

Most of the BM target radar feature extraction techniques are grounded on the uniformly and continuously sampled data in time domain, and some techniques such as HRRP and ISAR require wide band and high frequency sampled radar echoes. However, due to the practical demands on the BMWR, especially for phased array radars, which work in the mode of multi-task and multi-target, radar return for each target is usually segmented and even sparse in the time domain. This greatly increases the difficulties of the BM target feature extraction task. The analysis of the sparse signal from the BMWR is particularly important to detect and recognize non-cooperative unknown targets, especially for the BMD, a task that must be accomplished swiftly and with as few measurements as possible.

According to the electromagnetic scattering mechanism, in the high-frequency region, the signal returned from a target can be modeled approximately by a sum of signals scattered from some dominant and discrete radiation sources on the target, referred to as scattering centers [10], which implies that the radar signal from 
the $\mathrm{BM}$ in the high-frequency region is sparse. The scattering centers whose number is usually less than ten are normally associated with significant geometric features of the target. The relative position of the scattering centers is a key feature in the missile target recognition task.

Both the sparse nature of the scattering centers and the discontinuous availability of the target's radar return motivate the use of the sparse component analysis (SCA) technique for the extraction of the BM target features, such as the precession frequency and the scattering center relative locations.

So, aiming at identifying the special characteristics of the BM target returns from the ground based warning radar, a method of jointly estimating the precession frequency and the locations of the scattering centers is proposed in this article. In Section 2, the radar signal model of a conical warhead is analyzed and the measurement matrix for SCA is established; Section 3 presents the SCA method using the OMP algorithm to estimate the precession frequency and image the scattering centers of the BM. In order to reduce the computational requirement, the nonlinear least square (NLLS) algorithm is employed before the OMP processing to get a coarse estimate of the precession frequency. Simulation results are provided in Section 4 to assess the performance of the proposed method, and are followed by conclusions in Section 5.

\section{Signal model}

Precession is a motion unique to the BM in the midcourse phase. Research on the precession motion of the BM target in the United States goes back to the 1960 s and the feasibility of recognizing the real warhead and decoys based on the precession motion was validated in the two "Firefly" missions in 1990 [11]. A conical tip is a commonly seen feature in many ballistic missiles [12]. Figure 1 illustrates the precession motion model of a conical warhead. The warhead spins around its geometrical axis and precesses along the direction of velocity $\boldsymbol{v}$.

In order to analyze the radar return from the BM, we establish a Cartesian coordinate system with the origin point $O$ at the center of the BM bottom, set the geometrical axis as the $x$ axis, and set the $y$ axis vertical to the radar incident plane, as shown in Figure 2. The radar return from the BM target can be described as

$$
s(t)=\iint_{\Omega} \rho(x, y) \exp \left[-j 4 \pi f_{0} \frac{R_{O_{1}}(t)+\left(x+O O_{1}\right) \cos \varphi(t)+\gamma \sin \varphi(t)}{c}\right] d x d y
$$

where $f_{0}$ is the radar carrier frequency, $c$ is the speed of light, $\rho(x, y)$ is the scattering intensity at $(x, y)$ in the coordinate system, $\phi(t)$ is the aspect angle of the target,
$\Omega$ stands for the target space, $R_{O_{1}}(t)$ is the radial distance of the mass center $O_{1}$ from the transmitter, and $O O_{1}$ is the distance from the point $O_{1}$ to the bottom center $O$. During the mid-course phase (above the atmosphere), gravitation is the only force acting on the $\mathrm{BM}$, which means $R_{O_{1}}(t)$ can be calculated based on the two body motion theory [13].

According to the geometry and the precession model of a rigid body object, as illustrated in Figure 1, the relationship between the aspect angle $\phi(t)$, the precession angle $\theta(t)$, the precession frequency $f_{p}$, and the observation time $t$ can be expressed as

$$
\varphi(t)=\arccos \left\{\frac{\sin \theta(t) \sin \beta(t) \cos \left[2 \pi f_{p}\left(t_{0}+t\right)+\phi_{0}\right]}{\cos ^{2} \theta(t)}+\cos \theta(t) \cos \beta(t)-\frac{\sin ^{3} \theta(t) \sin \beta(t)}{\cos ^{2} \theta(t)}\right\}
$$

where $\varphi_{0}$ is the initial reference angle, $t_{0}$ is the initial reference time and $\beta(t)$ is the angle between the radar line of sight (LOS) and the vector direction of the warhead velocity $\boldsymbol{v}$. Compared with the aspect angle $\phi(t)$, $\theta(t)$, and $\beta(t)$ change very slowly. So it is not complicated to compensate for the time-variation of the parameters $\theta(t)$ and $\beta(t)$ and the actual method of the compensation [14] need not be discussed in this article. Therefore, one can infer that the aspect angle $\phi(t)$ is pseudo-periodic and the "period" $T_{p}$ is determined by the precession frequency $f_{p}$.

As we can see from (1), the radar scattering mechanisms are complicated, even for a geometrically simple target [15]. However, the concept of scattering centers provides a physically relevant, yet concise description of the object, and is thus a good candidate for use in radar signature modeling as well as target recognition [10]. According to the scattering center theory, (1) can be rewritten as [8]

$$
s(t)=\sum_{m=1}^{M} a\left(x_{m}, \gamma_{m}\right) \exp \left[-j 4 \pi f_{0} \frac{R_{O_{1}}(t) x_{m}^{\prime} \cos \varphi(t)+\gamma_{m}^{\prime} \sin \varphi(t)}{c}\right], t=\left[t_{1}, t_{2}, \ldots, t_{N}\right] .
$$

where $M$ is the number of all possible scattering centers on the area illuminated by the radar, $\left(x_{m}, y_{m}\right)$ is the coordinate of the $m$ th possible scattering center, and $a_{m}=a\left(x_{m}, y_{m}\right)$ represents the scattering coefficient. The positions of the possible scattering centers are chosen to be uniformly distributed in the covered area and their number $M$ is chosen according to the azimuth resolution of the radar, whose limit is $\lambda / 4$ [16], where $\lambda$ is the wavelength of the radar $\lambda=f_{0} / c$. The actual number of scattering centers is much smaller than $M$, which means that most of the $a_{m}$ 's are zero. Generally, the upside of the BM is full of the materials with low density, such as the fuze and some carbonaceous stuff, and the main load of the BM is at the bottom [14]. Thus, the distance between the mass center to the bottom center $O O_{1}$ is normally very small. And 


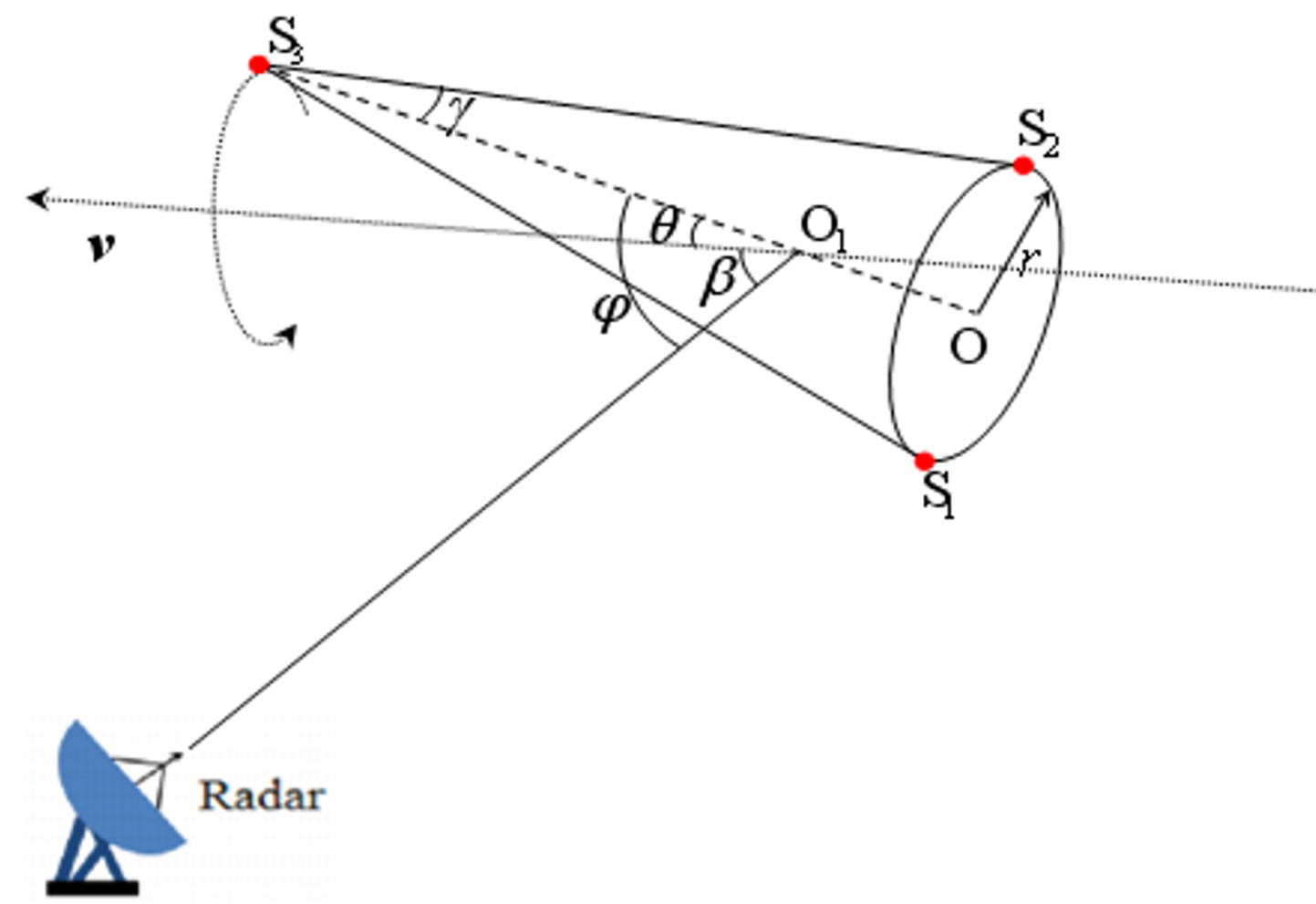

Figure 1 Precession motion of a conical warhead

the value of $O O_{1}$ does not affect the relative positions of the scattering centers on the BM. Hence, we set $x_{m}^{\prime}=x_{m}+O O_{1}$ and $y_{m}^{\prime}=y_{m}$. In (3), we define $\Phi_{m}\left(t, f_{p}\right)$ as the phase function of the $m$ th scattering center, which is

$$
\Phi_{m}\left(t, f_{p}\right)=4 \pi f_{0} \frac{\boldsymbol{R}_{O_{1}}(t)+x_{m}^{\prime} \cos \varphi(t)+y_{m}^{\prime} \sin \varphi(t)}{c} .
$$

Define

$$
\begin{aligned}
\boldsymbol{s}^{T} & =\left[\begin{array}{llll}
s\left(t_{1}\right) & s\left(t_{2}\right) & \cdots & s\left(t_{N}\right)
\end{array}\right] \\
\boldsymbol{a}^{T} & =\left[\begin{array}{llll}
a_{1} & a_{2} & \cdots & a_{M}
\end{array}\right] \\
\boldsymbol{Q} & =\left[\begin{array}{cccc}
e^{-j \boldsymbol{\Phi}_{1}\left(t_{1}, f_{p}\right)} & e^{-j \boldsymbol{\Phi}_{2}\left(t_{1}, f_{p}\right)} & \cdots & e^{-j \boldsymbol{\Phi}_{M}\left(t_{1}, f_{p}\right)} \\
& \ddots & & \vdots \\
& \ddots & \\
e^{-j \boldsymbol{\Phi}_{1}\left(t_{N}, f_{p}\right)} & e^{-j \boldsymbol{\Phi}_{2}\left(t_{N}, f_{p}\right)} & \cdots & e^{-j \boldsymbol{\Phi}_{M}\left(t_{N}, f_{p}\right)}
\end{array}\right]
\end{aligned}
$$

where $s \in \mathbb{C}^{N}$ is the observation vector, $\boldsymbol{a} \in \mathbb{C}^{N \times M}$ is the measurement matrix (dictionary) with unknown parameter $f_{p}$. Define $\Pi \triangleq\left\{\boldsymbol{a} \in \mathbb{C}^{M}: \boldsymbol{Q} \boldsymbol{a}=\mathbf{s}\right\}$. If there is $\boldsymbol{a} \in \boldsymbol{\Pi}$, then $\boldsymbol{a}$ is a representation of the signal $\boldsymbol{s}$ in the dictionary $\boldsymbol{Q}$. And if we have $\|\boldsymbol{a}\|_{0}<M$, then $\boldsymbol{a}$ is a sparse representation of the signal $s$, where $\|a\|_{0}=\operatorname{Card}\left\{j:\left|a_{j}\right|\right.$ $\neq 0\}$. Especially, if $\tilde{\boldsymbol{a}}=\arg \min _{\boldsymbol{a} \in \Pi}\|\boldsymbol{a}\|_{0}$, then $\tilde{\boldsymbol{a}}$ is the sparsest representation of the signal $s$, and $K=\|\tilde{a}\|_{0}$ is the sparsity.

For the conical missile as shown in Figure 1, there are three scattering centers on the target theoretically [17]: one at the top $S_{3}$, and two at the bottom of the BM $S_{1}$ and $S_{2}$. The distribution of the three scattering centers on the BM is shown in Figure 2. So, the sparsest representation $\tilde{\boldsymbol{a}}$ of the signal $\boldsymbol{s}$ has non-zero values only at the positions of $S_{1}, S_{2}$, and $S_{3}$, and the sparsity $K$ is the number of the scattering center, with $K=3$. Hence, if we can estimate the sparsest representation $\tilde{s}$, we can then image the BM target simply by calculating the non-zero value positions of the vector $\tilde{\boldsymbol{a}}$. The sparsest representation estimation $\tilde{\boldsymbol{a}}$ can be achieved by the following expression

$$
\min _{a \in \mathbb{C}^{M}}\|a\|_{0} \text { s.t. } Q a=s
$$

Assuming that the receiver noise is white Gaussian noise, the observation system is given by

$$
y=Q a+v
$$

where $\boldsymbol{y}=\left[y\left(t_{1}\right), \ldots, y\left(t_{N}\right)\right]^{T}$ is the observation vector, $\boldsymbol{v}$ $=\left[v\left(t_{1}\right), \ldots, v\left(t_{N}\right)\right]^{T}$ is the receiver noise vector which is 


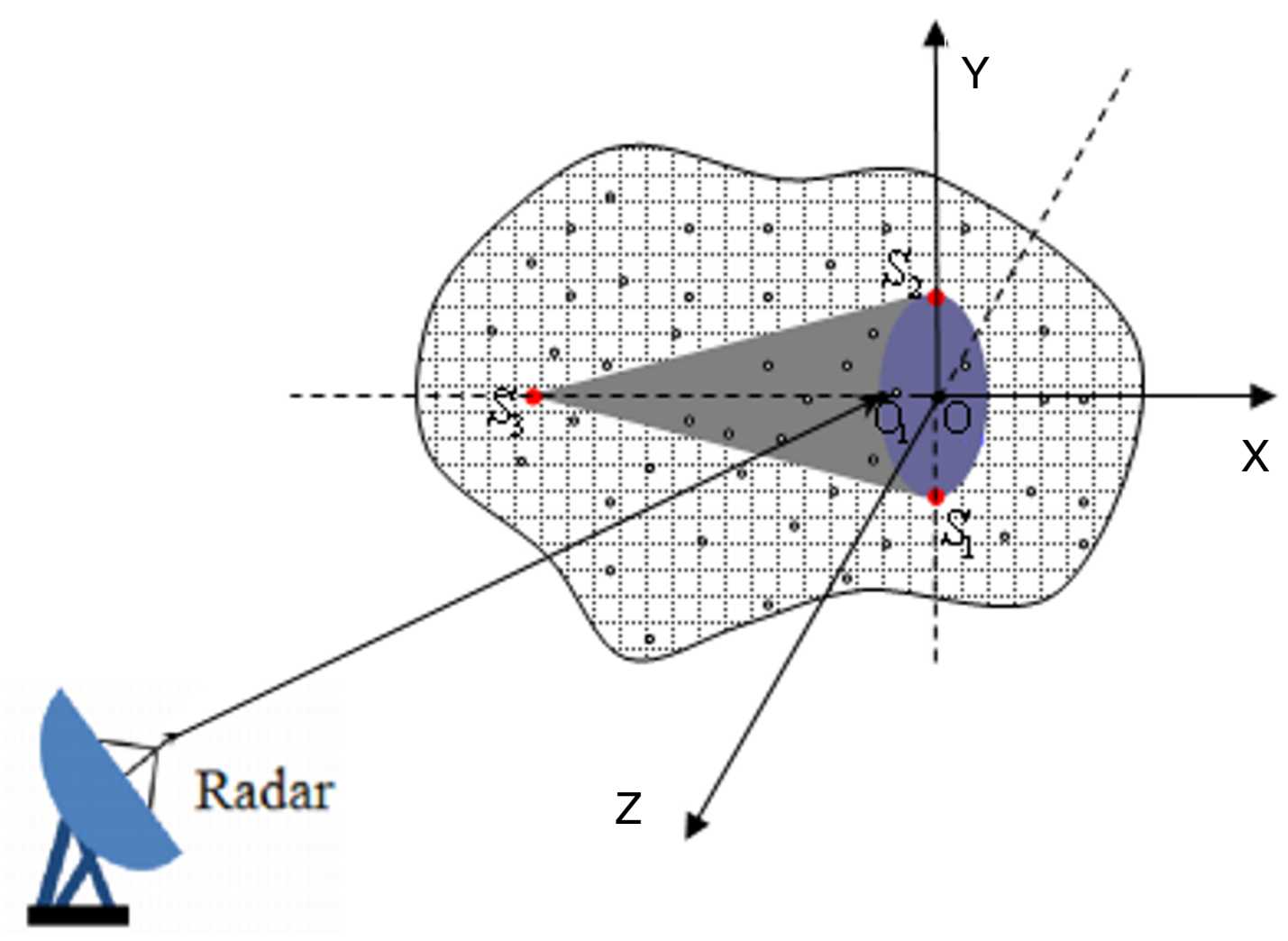

Figure 2 Distribution of the BM scattering centers.

modeled as a zero-mean Gaussian vector with covariance matrix $\sigma^{2} I_{N \times N}$ with $I_{N \times N}$ denoting the $(N \times N)$ identity matrix and $\sigma^{2}$ denoting the noise variance. So taking the receiver noise into account, (6) is reformulated as

$$
\min _{a \in \mathbb{C}^{M}}\|\boldsymbol{a}\|_{0} \text { s.t. }\|y-Q a\|_{2} \leq \sigma
$$

Solving (6) or (8) with the $\ell_{0}$ norm is both numerically unstable and NP-hard [18]. Fortunately, optimization algorithms, such as the basic matching pursuit (MP) [19] and orthogonal matching pursuit (OMP) [20], can exactly recover sparse signals with high probability.

\section{Precession frequency estimation and BM imaging}

As it has been discussed above, the SCA is suitable to process the radar signal from a $\mathrm{BM}$ which is sparse. Thus, the task of BM target imaging, i.e., estimating the positions of the scattering centers on the BM, can be carried out by SCA based on the non-uniformly or even sparsely sampled radar data, which can significantly save the time resource in the BMD system and satisfy the special working mode of the BMWR. However, as mentioned in the sparse system model in (8), there is an unknown parameter $f_{p}$ in the measurement matrix $Q$ that has to be estimated. Further, the precession frequency $f_{p}$ is also an important feature parameter in the $\mathrm{BM}$ target recognition.

Here, we propose to jointly estimate the positions of the scattering centers and the precession frequency. The proposed SCA based method consists of solving the OMP for each precession frequency candidate and retain the solution which minimizes the mean square error (MSE) between the measurements and estimated signal. In order to reduce the search space for $f_{p}$ and thus reduce the computational burden of the system, we also propose to initialize the estimation of $f_{p}$ by estimating the period of the observed signal using the NLLS. The NLLS is a widely used estimation approach since it makes no assumption on the distribution of the noise [21]. However, in our problem, the accuracy of the NLLS is limited by the sparse measurements, and thus the NLLS can only be used as an initial guess and a more accurate estimate has to be achieved in the following SCA process. 


\subsection{NLLS estimation of the precession frequency}

Assuming $\beta(t)$ and $\theta(t)$ to be constant during the observation time, the aspect angle in (2) can be rewritten as

$$
\phi(t)=\arccos \left(w_{1} \cos \left[2 \pi f_{p}\left(t+t_{0}\right)+\phi_{0}\right]+w_{2}\right)
$$

where, $w_{1}$ and $w_{2}$ are constants, given by $w_{2}=\cos \theta \cos \beta-\frac{\sin \theta^{3} \sin \beta}{\cos \theta^{2}}$,

$w_{2}=\cos \theta \cos \beta-\frac{\sin \theta^{3} \sin \beta}{\cos \theta^{2}}$. The aspect angle is thus periodic with period $T_{p}=1 / f_{p}$ and so are the $\Phi_{m}\left(t, f_{p}\right)^{\prime} \mathrm{s}$ and the signal $s(t)$ in (3).

The estimation of all unknown parameters using the maximum likelihood (ML) approach would require a highly nonlinear and multi-dimensional optimization. However, if one is interested in estimating the precession frequency $f_{p}$ only, a suboptimum but computationally attractive approach is described next.

The received signal can be regarded as a periodic signal of an unknown shape in AWGN. If the sampling is very fine, then the period can be estimated easily using time-domain autocorrelation. Otherwise, one has to resort to the frequency domain, as follows.

Using the Fourier series analysis, the received signal can be expressed as

$$
y(t)=\sum_{g=-\infty}^{\infty} c_{g} e^{-j 2 \pi g f_{0} t}+v(t), t=t_{1}, t_{2}, \ldots, t_{N} .
$$

To estimate the parameter $f_{p}$, we first truncate the above summation to $2 G+1(\ll N)$ terms, which yields

$$
y(t) \approx \sum_{g=-G}^{g=G} c_{g} e^{-j 2 \pi g f_{p} t}+v(t), t=t_{1}, t_{2}, \ldots, t_{N} .
$$

and then solve the following NLLS problem

$$
\left(\hat{\boldsymbol{c}}, \hat{f}_{p}^{0}\right)=\arg \min _{c, f} \sum_{t=t_{1}}^{t_{N}}\left|y\left(t_{n}\right)-\sum_{g=-G}^{G} c_{g} e^{-j 2 \pi g f t_{n}}\right|^{2}
$$

The precession frequency can thus be estimated by

$$
\hat{f}_{p}^{0}=\arg \max _{f} \gamma^{H} \Gamma_{f}\left(\Gamma_{f}^{H} \Gamma_{f}\right)^{-1} \Gamma_{f}^{H} \boldsymbol{y}
$$

where

$$
\begin{aligned}
& \gamma^{T}=\left[\gamma\left(t_{1}\right) y\left(t_{2}\right) \cdots \gamma\left(t_{N}\right)\right] \\
& \boldsymbol{c}^{T}=\left[\begin{array}{llll}
c_{G} & c_{G-1} & \cdots & c_{-G}
\end{array}\right]
\end{aligned}
$$

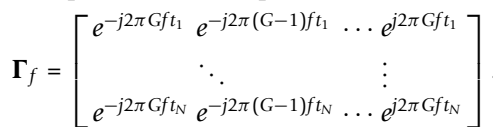

In (13), parameter $G$ should be designed to provide a good trade-off between modeling accuracy (bias) and estimation variance. Indeed, modeling accuracy increases with $G$ (so the bias decreases), but when $G$ increases the number of unknown parameters to estimate increases and this leads to a higher estimation variance. This is a well known problem in estimation theory, and thus will not be discussed here. In our simulation setup, $G=64$ was shown to give good results.

\subsection{BM target imaging based on SCA}

The OMP, a powerful and efficient algorithm for sparse signal recovery [20], is a greedy algorithm similar to the basic MP algorithm. The general goal of this technique is to obtain a sparse signal representation by choosing, at each iteration, a dictionary atom that is best adapted to approximate part of the signal. At each iteration, the OMP approach gives rise to the set of coefficients yielding the linear expansion that minimizes the distance to the signal.

Let $\boldsymbol{q}_{k}$ denote the $k$ th column vector of matrix $\boldsymbol{Q}$. For the sparse signal model in (8), let $\boldsymbol{b}_{p}$ be the $p^{\text {th }}$ order residue and initialize the residual $\boldsymbol{b}_{0}=\boldsymbol{y}$. The indices of the $p$ vectors selected are stored in the index vector $\boldsymbol{I}_{p}=$ $\left[k_{1}, k_{2}, \ldots, k_{p}\right]$, and the vectors are stored as the columns of the matrix $\Omega_{p}=\left[q_{k_{1}}, q_{k_{2}}, \ldots, q_{k_{p}}\right]$. The OMP algorithm selects $k_{p}$ at the $p$ th iteration by finding the vector best aligned with the residual obtained by projecting $\boldsymbol{b}_{p}$ onto the dictionary components, that is

$$
k_{p}=\arg \max _{l}\left|<\boldsymbol{q}_{1}, \boldsymbol{b}_{p}>\right|, \quad l \notin \boldsymbol{I}_{p-1}
$$

where $\left\langle\boldsymbol{q}_{l}, \boldsymbol{b}_{p}\right\rangle$ means the inner product of vectors $\boldsymbol{q}_{l}$ and $\boldsymbol{b}_{p}$. The re-selection problem is avoided with the stored dictionary. The selected vector component $\boldsymbol{q}_{k_{p}}$ is orthogonalized by the Gram-Schmidt algorithm as

$$
\boldsymbol{u}_{p}=\boldsymbol{q}_{k_{p}}-\sum_{l=0}^{p-1} \frac{<\boldsymbol{q}_{k_{p}} \boldsymbol{u}_{l}>}{\left\|\boldsymbol{u}_{l}\right\|_{2}^{2}} \boldsymbol{u}_{l}
$$

The residual $\boldsymbol{b}_{p}$ is updated as

$$
\boldsymbol{b}_{p+1}=\boldsymbol{b}_{p}-\frac{<\boldsymbol{b}_{p}, \boldsymbol{u}_{p}>}{\left\|\boldsymbol{u}_{p}\right\|_{2}^{2}} \boldsymbol{u}_{p}
$$

The algorithm terminates when $\left\|\boldsymbol{b}_{p+1}\right\|_{2} \leq \sigma$.

Since there is an unknown parameter $f_{p}$ in the measurement matrix $\boldsymbol{Q}$, we perform the OMP algorithm for each candidate for $f_{p}$ and retain the candidate which minimizes the mean squares error between the corresponding sparse representation and observation vector, i.e. 


$$
\hat{f}_{p}=\arg \min _{f \in \Upsilon}\left\|y-\hat{Q}_{f} \hat{\boldsymbol{a}}_{f}\right\|_{2}
$$

where $\hat{\boldsymbol{a}}_{f}$ is the sparse representation obtained by the OMP algorithm for the frequency candidate $f \in \Upsilon$, and the search range $\Upsilon$ is chosen to be centered around the NLLS estimate $\hat{f}_{p}^{0}$ presented in section 3.1.

Therefore, the proposed SCA method is summarized as follows.

Step 1: Obtain the initial estimate of the precession frequency $\hat{f}_{p}^{0}$ using the NLLS method;

Step 2: Set the search range $\Upsilon$ as $\Upsilon \equiv\left(\hat{f}_{p}^{0}-\varepsilon_{L}, \hat{f}_{p}^{0}+\varepsilon_{P}\right)$; for every $f \in \Upsilon$, obtain a sparse representation $\hat{\boldsymbol{a}}_{f}$ via the OMP algorithm;

Step 3: Obtain the estimate $\hat{f}_{p}$ of the precession frequency and the corresponding sparsest representation $\hat{a}_{\hat{f}_{p}}$ using eq. (18).

Step 4: Calculate the sparsity as $\hat{\boldsymbol{K}}=\left\|\hat{\boldsymbol{a}}_{\hat{f}_{p}}\right\|_{0}$.

\section{Simulation and experimental results}

When the BMWR is working in the mode of multi-target and multi-task detection, the radar return for each target is non-uniformly sampled and time-segmented. The trajectory (see Figure 3) was calculated based upon the two-body motion theory [13]. The geographic coordinate values of the $B M$ launch point are $(125.19 E, 43.54 N, 0)$, the fall point is $(110.20 E, 20.02 N$, $100)$ and the ground based radar sat is $(119.58 E, 31.47 N, 50)$. We assume that there are four observation time segments of the BM, which begin at the 650th, 651th, 652th and the 652.5th second after the $\mathrm{BM}$ is launched, respectively. For simplicity, the observation time segments are set to be the same and equal to 50 milliseconds, and the pulse repetition frequency (PRF) of each segment is set to $f_{s}=2048 \mathrm{~Hz}$. Note that in practice, the observation durations and the PRF of the different segments may be set to be different.

The returned signal is from a ground based BMWR with carrier frequency $f_{0}=5.0 \mathrm{GHz}$. The simulated $\mathrm{BM}$ size considered in this article was set by reference to the

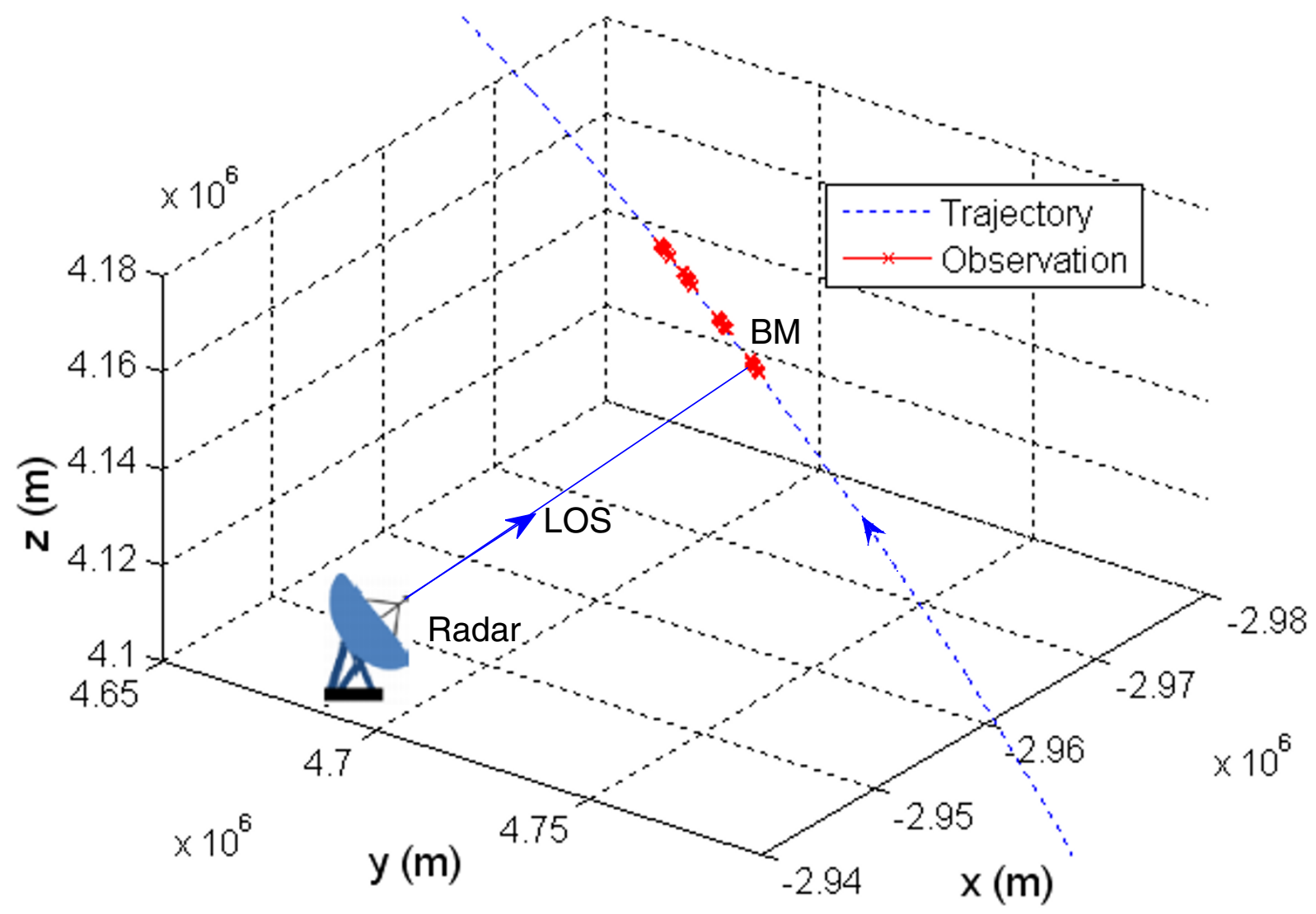

Figure $3 \mathrm{BM}$ trajectory and time segmented observation of the BMWR. 


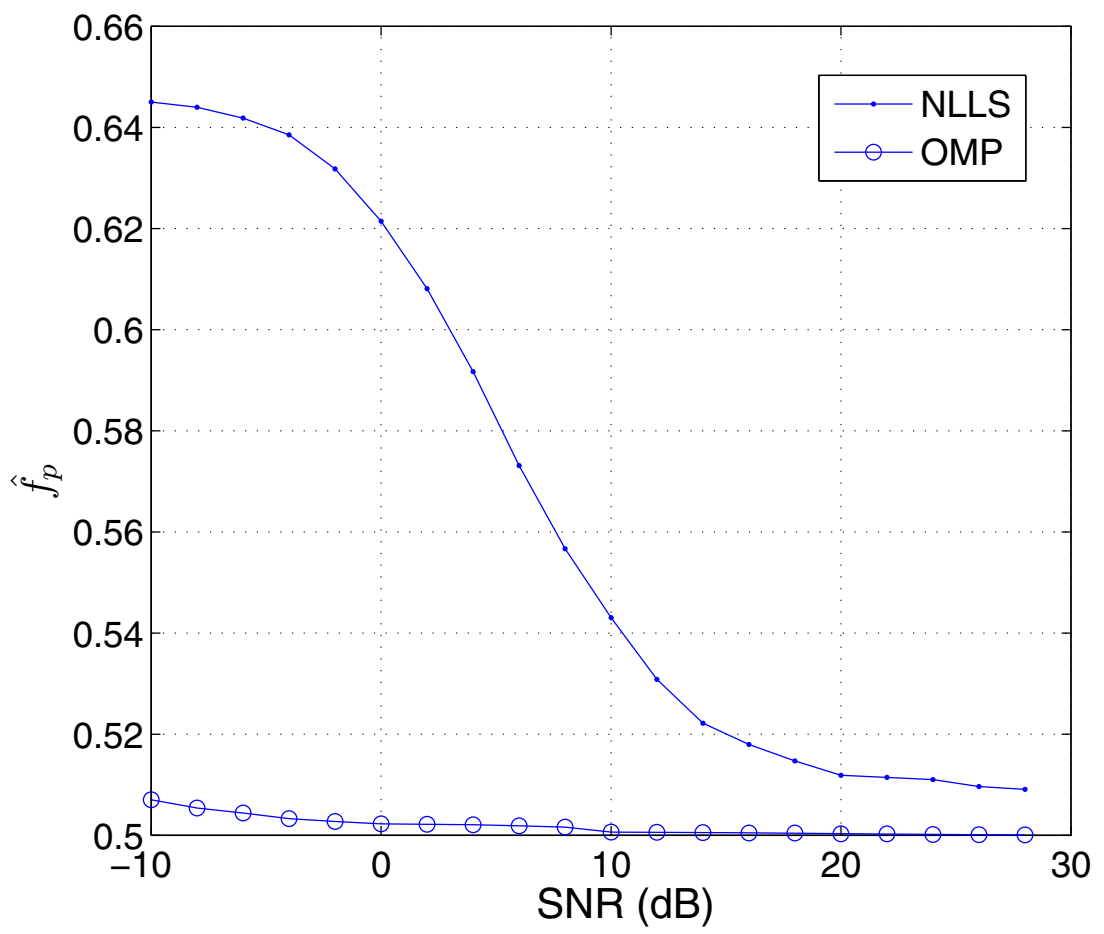

Figure 4 Plots of mean estimation of $f_{p}$ using NLLS and OMP versus SNR.

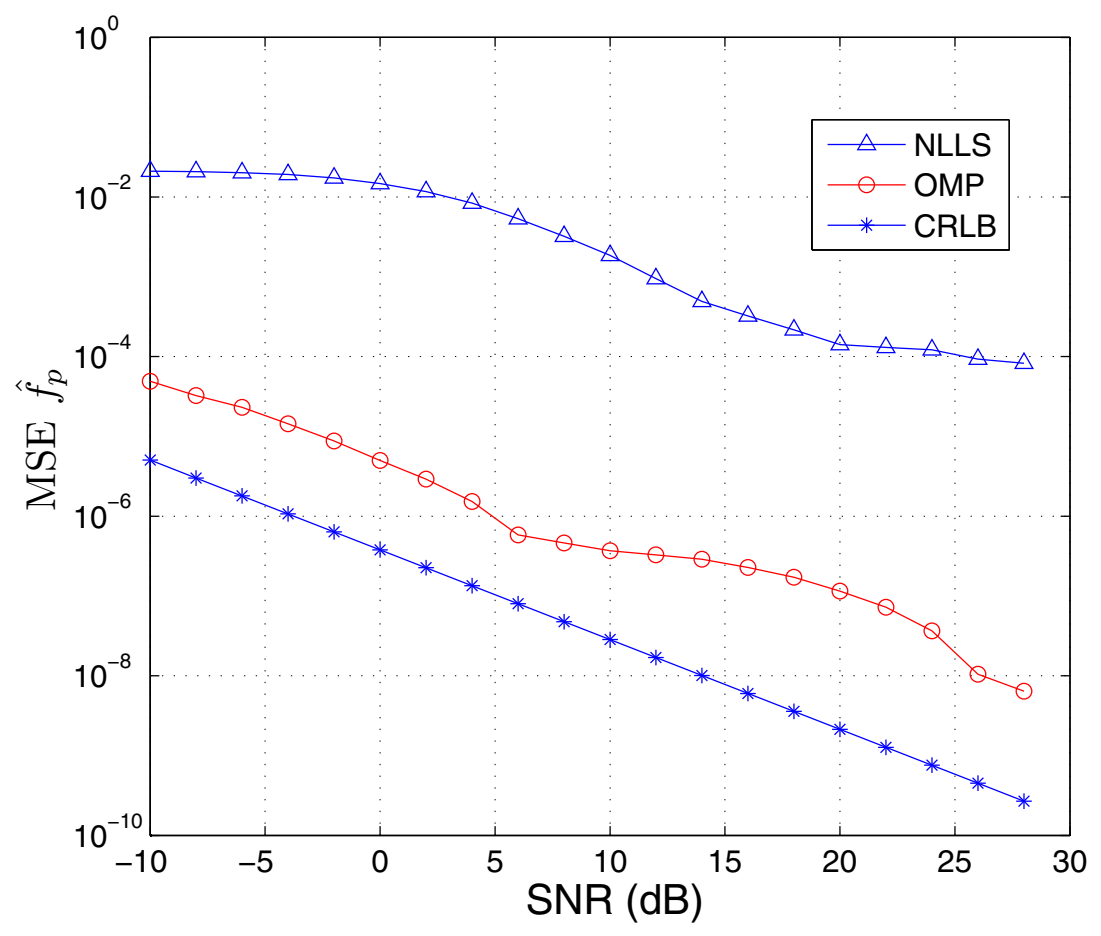

Figure 5 Plots of mean square estimation error for $f_{p}$ using NLLS and OMP versus SNR. 


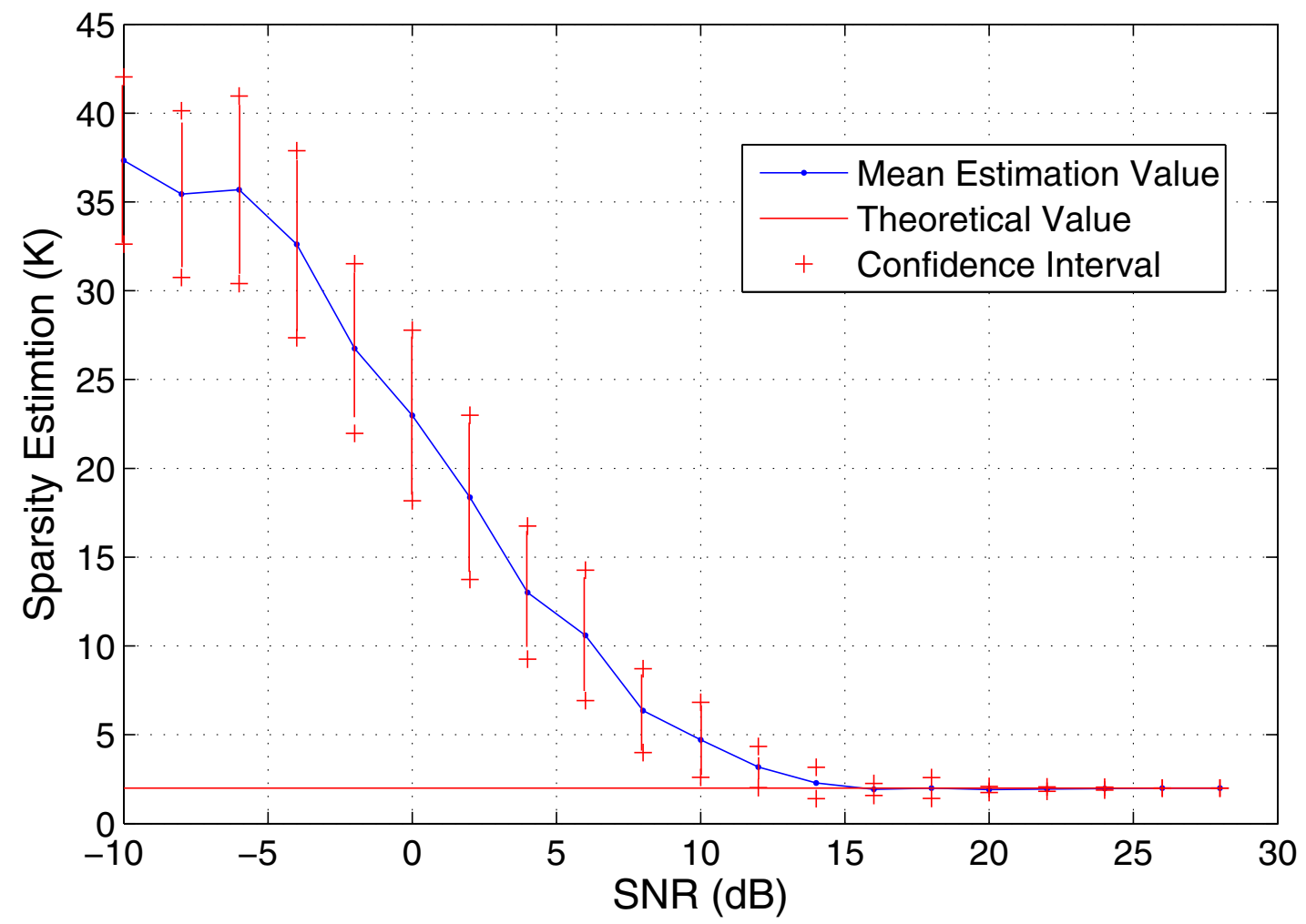

Figure 6 Mean and confidence interval of sparsity $K$ estimation versus SNR.

Indian Agni-II BM [22]: the length of the warhead is $H$ $=2.09 \mathrm{~m}$ and the bottom radius is $r=0.329 \mathrm{~m}$. We assume that the target area is $2.5 \mathrm{~m} \times 1 \mathrm{~m}$, as shown in Figure 2. Taking into account the target recognition requirements and the practical radar resolution capability, the target area is uniformly divided into $60 \times 60$ small rectangles along the coordinate axes, which means that the number of possible scattering centers $M$ in (3) is set as $M=360$, the resolution is about $\lambda / 2$ along the $x$ axis and $\lambda / 4$ along the $y$ axis.

Figure 4 shows the plots of the mean estimation of $f_{p}$ using NLLS and OMP methods versus SNR. Figure 5 shows the plots of the MSE of the estimation of $f_{p}$ estimation for the two methods. As a benchmark, the plot of the Cramér-Rao lower bound (CRLB) versus SNR is also displayed in Figure 5. For the parameter estimation using OMP, the step size of the discrete grid for estimating $f_{p}$ at every SNR was set to be lower than the square root of the CRLB. As shown in Figure 5, the MSE of the OMP estimation of $f_{p}$ is two orders of magnitude lower than that of the NLLS estimation, which means that with the prior information of the sparsity of the scattering centers, the SCA method using the OMP algorithm can achieve significantly better estimation performance. With SNRs higher than 10dB, the MSE of the OMP-based frequency estimate is lower than $10^{-}$; this accuracy is good enough for the scattering center imaging process, as shown in Figures 6 and 7.

Figure 6 displays the mean values and confidence intervals of the sparsity (K) estimate versus SNR. When the SNR is higher than $15 \mathrm{~dB}$, the mean value of the sparsity estimate is two. According to the theoretical analysis in Section 2, the number of scattering centers on the BM is three. However in the geometric model of the $\mathrm{BM}$ in this article, the radius of the conical BM top is set to zero and thus the intensity (which is determined by the BM top radius) of the scattering center $S$ ${ }_{3}$ is zero too. Hence, the actual value of the sparsity is $K$ $=2$, and the simulation result is in agreement with the simulation setting.

With the estimation of the sparse representation $\hat{\boldsymbol{a}}$, the target scattering centers can be easily imaged according to the Cartesian coordinate system in Figure 2. Figure 7 displays the BM target scattering center radar images with different SNRs. As shown in Figure 7, the estimation of $\hat{\boldsymbol{a}}$ is accurate when the SNR is higher than $15 \mathrm{~dB}$, in terms of both the positions and the number of the scattering centers. 

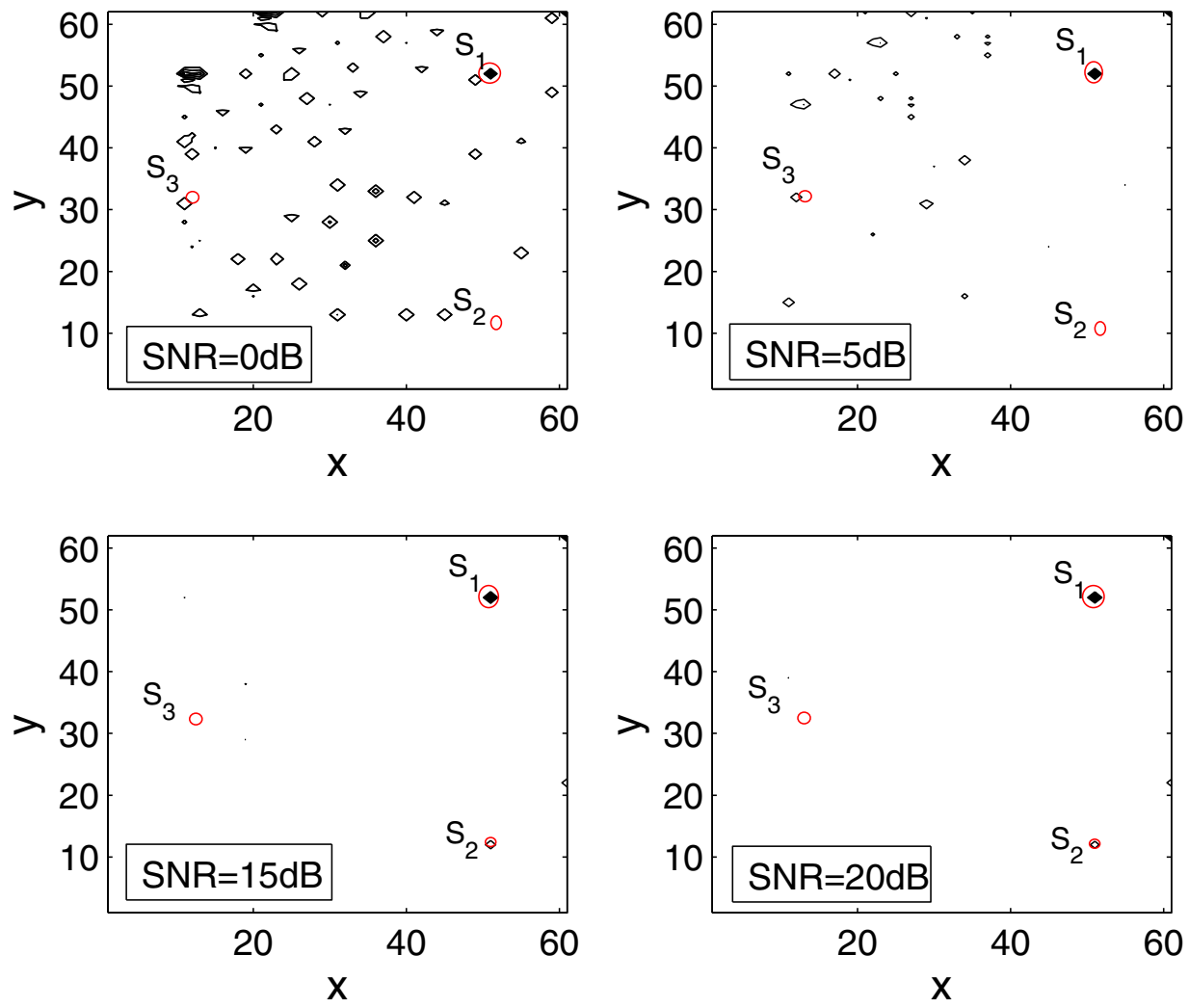

Figure 7 BM target scattering center radar images with different SNRs

\section{Conclusion}

The micro-Doppler parameter estimation and scattering center imaging are very important in the BM target recognition system. According to the electromagnetic scattering mechanism, the SCA technique based on the sparse measurements from the BMWR can solve this task efficiently and can also greatly save the time resources in the BMD system. The article analyzed the BM radar signal model and established the sparse dictionary according to the sparse nature of the scattering centers of the BM. A method based on the NLLS and the OMP algorithms was proposed to estimate the precession frequency and image the BM scattering centers. The proposed method can extract these feature parameters with limited radar measurements.

\section{Acknowledgements}

This study was supported by the National Science Foundation of China under grant 61002021

\section{Author details}

${ }^{1}$ College of Electronic Science and Engineering, National University of Defense Technology, Changsha, Hunan 410073, P.R. China ${ }^{2}$ School of
Electronic and Electrical Engineering, The University of Leeds, Leeds, UK ${ }^{3}$ International University of Rabat, Rabat, Morocco

\section{Authors' contributions}

LL carried out the theoretics analysis of using sparse component analysis on radar measurements, performed the simulation experiments and drafted the manuscript. XD participated in its design and manuscript drafting. MG involved in the design of the study and revised it critically for important intellectual content of the manuscript. WH conceived of the study and gave the final approval of the version to be published. DM participated in the proof reading and the theory analysis. All authors read and approved the final manuscript.

\section{Competing interests}

The authors declare that they have no competing interests.

Received: 8 September 2011 Accepted: 9 February 2012

Published: 9 February 2012

\section{References}

1. RH Chen, JL Speyery, Terminal and boost phase intercept of ballistic missile defense, in AlAA Guidance, Navigation and Control Conference and Exhibit, AIAA, 2008-6492 (2008)

2. RJ Cepek, Ground-based midcourse defense: continue testing, but operational fielding must take a backseat to theater missile defense and homeland security, in Joint Forces Staff College, Joint Advanced Warfighting School (2005)

3. R Elias, JV Nevarez, Active Nutation adn Precession Control for Exoatomospheric Spinning Ballistic Missiles, in AIAA Guidance, Navigation and Control Conference and Exhibit, AIAA, 2008-6998 (2008) 
4. VC Chen, F Li, S Ho, H Wechsler, Analysis of Micro-Doppler Signatures. IEE Proc Rdadar Sonar Navigat. 150(4), 271-276 (2003). doi:10.1049/iprsn:20030743

5. VC Chen, F Li, S Ho, H Wechsler, Micro-Doppler effect in radar: phenomenon, model, and simulation study. IEEE Trans Aerospace Electron Syst. 42(1), 2-21 (2006). doi:10.1109/TAES.2006.1603402

6. H Gao, L Xie, S Wen, K Yong, Research on precession of ballistic missile warhead based on micro-Doppler analysis. Syst Eng Electron. 30(1), 50-52 (2008)

7. ME Clark, High range resolution techniques for Ballistic missile, in British Aerospace Land and Sea Systems, 1-6 (1999)

8. X Xu, R Narayanan, Three-dimensional interferometric ISAR imaging for target scattering diagnosis and modeling. IEEE Trans Image Process. 10(7), 1094-1102 (2001). doi:10.1109/83.931103

9. Q Wang, M Xing, G Lu, High-resolution three-dimensional radar imaging for rapidly spinning targets. IEEE Trans Geosci Remote Sens. 46(1), 22-33 (2008)

10. M Gerry, L Potter, I Gupta, A Van Der Merwe, A parametric model for synthetic aperture radar measurements. IEEE Trans Antennas Propagat. 47(7), 1179-1188 (1999). doi:10.1109/8.785750

11. K Schultz, S Davidson, A Stein, J Parker, Range Doppler laser radar for midcourse discrimination-the firefly experiments, in AIAA and SDIO, Annual Interceptor Technology Conference, vol. 2. Albuquerque, NM, 6-9 (1993)

12. SH Lee, Investigation of the effects of target feature variations on ballistic missile RCS, in Air Force Institute of Technology, Graduate School of Engineering and Management (2006)

13. E Stiefel, G Scheifele, Linear and regular celestial mechanics. Perturbed twobody motion, in Numerical Methods Canonical Theory (1975)

14. K Li, W Jiang, Y Liu, Feature extraction of cone with precession based on micro-Doppler, in IET International Radar Conference, Gui Lin, China, 1-5 (2009)

15. KT Kim, HT Kim, Two-dimensional scattering center extraction based on multiple elastic modules network. IEEE Trans Antennas Propagat. 51(4), 848-861 (2003). doi:10.1109/TAP.2003.811107

16. J Morris Swiger, Resolution limits of ultra wideband synthetic aperture radar using a rectangular aperture for FFT processing. IEEE Trans Aerospace Electron Syst. 30(3), 935-938 (1994). doi:10.1109/7.303768

17. P Huang, $\mathrm{H}$ Yin, $X \mathrm{Xu}$, Radar Target Characteristics, Publishing House of Electronics Industry, Beijing (2005)

18. T Blu, P Dragotti, M Vetterli, P Marziliano, L Coulot, Sparse sampling of signal innovations. IEEE Signal Process Mag. 25(2), 31-40 (2008)

19. SS Chen, DL Donoho, MA Saunders, Atomic decomposition by basic pursuit. SIAM J Sci Comput. 20(1), 33-61 (1998). doi:10.1137/ S1064827596304010

20. JA Tropp, AC Gilber, Signal recovery from random measurements via orthogonal matching pursuit. IEEE Trans Inf Theory 53(12), 4655-4666 (2007)

21. M Ghogho, A Swami, A Nandi, Nonlinear least squares estimation for harmonics in multiplicative and additive noise. Elsevier Signal Process. 78(1), 43-60 (1999)

22. A Vishwakarma, Agni-strategic Ballistic Missile, http://www.bharat-rakshak com/land-forces/Equipment/Others/379-Agni-IRB M-(Page-1).html (2008)

doi:10.1186/1687-6180-2012-24

Cite this article as: Liu et al:: Precession missile feature extraction using sparse component analysis of radar measurements. EURASIP Journal on Advances in Signal Processing 2012 2012:24.

\section{Submit your manuscript to a SpringerOpen ${ }^{\circ}$ journal and benefit from:}

- Convenient online submission

- Rigorous peer review

- Immediate publication on acceptance

- Open access: articles freely available online

- High visibility within the field

- Retaining the copyright to your article

Submit your next manuscript at $\gg$ springeropen.com 